\title{
Différenciation et marginalisation de la paysannerie en Sierra Leone
}

Differentiation and marginalisation of the peasantry in Sierra Leone

\section{Augustin Palliere}

\section{(2) OpenEdition}

\section{Journals}

\section{Édition électronique}

URL : http://journals.openedition.org/economierurale/4648

DOI : 10.4000/economierurale.4648

ISSN : 2105-2581

\section{Éditeur}

Société Française d'Économie Rurale (SFER)

\section{Édition imprimée}

Date de publication : 30 juin 2015

Pagination : 39-57

ISSN : 0013-0559

\section{Référence électronique}

Augustin Palliere, "Différenciation et marginalisation de la paysannerie en Sierra Leone », Économie rurale [En ligne], 347 | Mai-juin 2015, mis en ligne le 30 juin 2017, consulté le 02 mai 2019. URL : http:// journals.openedition.org/economierurale/4648; DOI : 10.4000/economierurale.4648 


\title{
Différenciation et marginalisation de la paysannerie en Sierra Leone
}

\author{
Augustin PALLIERE • AgroParisTech, UMR Prodig, Université Paris Ouest Nanterre \\ La Défense, UMR LAVUE \\ augustin.palliere@gmail.com.
}

\begin{abstract}
Dans le Nord de la Sierra Leone, région marginalisée sur le plan économique, les systèmes de production ne semblent pas avoir connu de différenciation, que ce soit avec la capitalisation ou par la spécialisation. Mais, dans la région étudiée, l'éclatement des unités de production/consommation et la monétarisation du revenu des producteurs sont à l'origine de l'émergence d'échanges marchands de force de travail au sein des villages. Ces échanges se traduisent par des disparités importantes, pour une agriculture manuelle, du point de vue du revenu agricole. Mais ces disparités ne se traduisent pas par une différenciation durable du fait de l'hétérogénéité du rapport consommateur/ actif, elle-même le résultat de l'intense circulation enfantine dans cette société paysanne.
\end{abstract}

MOTS-CLÉS : différenciation, marchandisation, force de travail, Sierra Leone

\section{Differentiation and marginalisation of the peasantry in Sierra Leone}

The northern Sierra Leone is an economically marginalized region. Production systems are not differentiated either in term of capital accumulation or specialization. In the studied region, the commodification of the labour force is explained both by production units split and monetization of the peasant income. Exchanges of labour force among the village account for the high level of agricultural income disparity, especially in a manual agriculture. But these disparities do not result into an enduring differentiation among the peasantry because of consumer/producer ratio heterogeneity, linked it self of the persistence of the children fosterage nowadays. (JEL Q19, 013).

KEYWORD: differentiation, commodification, labour force, Sierra Leone

$\mathbf{P}$ our Mazoyer et Roudart (1997), la « crise des paysanneries pauvres » à très faible niveau de capital est d'abord le résultat de leur mise en concurrence avec des agricultures beaucoup plus performantes. La Sierra Leone offre un exemple frappant de cette marginalisation : $58 \%$ des Sierra-Léonais sont des ruraux mais $30 \%$ de la population souffrent de sous-nutrition (FAO). L'agriculture est restée strictement manuelle - 1 tracteur en usage pour 12000 agriculteurs et la traction attelée est presque inexistante - et l'emploi d'intrants (phytosanitaires, engrais) d'origine industrielle est très peu développé - $0,3 \mathrm{~kg} / \mathrm{ha}$ d'engrais de synthèse en moyenne ${ }^{1}$. De

1. Toutes les données de cette introduction sont tirées de la base de données de la FAO : faostat. fao.org. longue date, la politique économique a favorisé le secteur minier au détriment du secteur agricole en facilitant les importations massives de riz à bas coût (Richards, 1996). La Sierra Leone n'a pas connu le développement, comme dans certains pays voisins, d'une économie de plantation grâce à l'afflux de migrants. Cette marginalisation sur les marchés internationaux est-elle généralisée à l'échelle locale des sociétés villageoises ? Malgré la faiblesse des niveaux de capital dans l'agriculture paysanne, des processus de différenciation entre producteurs sont-ils à l'œuvre ?

Dans les sources officielles (gouvernement, bailleurs), « l'attachement des paysans à l'agriculture de subsistance » est considéré comme la contrainte majeure pour la croissance agricole. Cette 
hypothèse guide la construction des typologies proposées : les advanced smallholders (petits producteurs modernes), supposés être market-oriented (orientés vers le marché), montreraient la voie aux rural poor smallholders (petits producteurs pauvres), considérés comme des (semi)-subsistence farmers (agriculteurs de subsistance) (MAFFS, 2009 ; MAFFS, 2010 ; Gomez y Paloma et al., 2012). Ce diagnostic vise à justifier une politique qui fait de l' «intégration au marché » et de la promotion du « secteur privé », c'està-dire des exploitations agro-industrielles financées par des capitaux étrangers, les priorités. Mais pour appréhender la différenciation à l'échelle locale, autarcie et marché, comme tradition et modernité, ne sont pas des catégories opérantes.

En Sierra Leone, les études conduites à l'échelle locale dans le sillon des livelihoods studies (études sur les moyens d'existence) (Chambers et Conway, 1992) proposent une vue beaucoup plus riche de la complexité des stratégies paysannes (Richards, 1985 ; Leach, 1994 ; Maconachie et Binns, 2007). Mais ces études, attentives à l'adaptation des pratiques aux conditions agro-écologiques ou socio-économiques à l'échelle locale, n'intègrent pas les dynamiques de long terme et les processus globaux (Scoones, 2009) qui affectent pourtant lourdement les sociétés paysannes.

La combinaison des échelles d'observation et d'analyse est justement au cœur du concept de système agraire tel qu'il est mobilisé en agriculture comparée (Cochet, 2011). Dans ce cadre, l'identification des trajectoires de différenciation des systèmes de production ${ }^{2}$ repose sur l'analyse

2. « [...] représentation modélisée d'un type d'unité de production possédant la même gamme de ressources, placées dans des conditions socioéconomiques comparables et qui pratiquent une même combinaison de production, bref un ensemble d'exploitations pouvant être représenté par un même modèle » (Cochet, 2011). des processus historiques d'accumulation en capital et de spécialisation (Cochet et Devienne, 2006). Mais de telles trajectoires peuvent-elles être identifiées dans des régions où l'agriculture est restée à très faible niveau de capital ? Comment construire des typologies de systèmes de production pertinentes quand la combinaison des activités productives diverses - vivrière et commerciale, agricoles et extra-agricoles - est similaire pour tous les producteurs? Associer l'approche systémique développée en agriculture comparée et les concepts de l'anthropologie économique marxiste (Paul, 2003) permet d'élargir le champ de la recherche et d'appréhender d'autres modalités d'accumulation et de différenciation. On propose d'articuler, à l'échelle d'une petite région, l'analyse historique de l'évolution des rapports sociaux et l'évaluation des performances technico-économiques des unités de production/consommation actuelles pour étudier la disparité et les processus de différenciation entre producteurs. Les résultats présentés dans cet article sont issus d'une recherche menée en plusieurs phases d'enquête entre 2007 et 2011 dans le chiefdom $^{3}$ de Sella Limba au nord du pays ${ }^{4}$.

Après avoir explicité notre approche, présenté le matériel empirique sur lequel reposent nos analyses et introduit la région étudiée, nous montrons, tout d'abord, comment les dynamiques agraires locales ont été lourdement infléchies par l'intégration économique de la région aux marchés nationaux et mondiaux. Nous insistons

3. Subdivision administrative en Sierra Leone, plusieurs chiefdoms constituent un district. La Sella Limba est un chiefdom de $400 \mathrm{~km}^{2}$ appartenant au district de Bombali.

4. Cette recherche a d'abord été réalisée dans le cadre de deux diagnostics agraires (Jolivel et al., 2007 ; Palliere, 2008) puis dans celui d'une thèse de doctorat soutenue le 2 juillet 2014 à l'Université Paris Ouest Nanterre La Défense (Palliere, 2014). 
notamment sur le processus de marchandisation de la force de travail résultant de l'éclatement des groupes domestiques conjugué à la monétarisation du revenu des producteurs. Aujourd'hui, l'accès à la force de travail aux moments clés du calendrier agricole est au cœur des rapports sociaux qui se nouent au sein de la paysannerie à l'occasion des processus de production. C'est sur la base de ce résultat que nous évaluons, ensuite, les performances économiques des unités de production/ consommation pour mesurer les disparités actuelles entre producteurs et interroger les processus de différenciation au sein de la paysannerie.

\section{Approche, méthode et sources pour l'étude des dynamiques agraires en Sella Limba}

Dans Femmes, greniers et capitaux, Meillassoux (1975) propose d'analyser ensemble les rapports de production - partage des moyens de production - et de reproduction, notamment les relations matrimoniales. Les premiers assurent la survie de la « communauté domestique » à court terme, d'un cycle agricole à l'autre ; les seconds, sa perpétuation sur le long terme, d'une génération à l'autre (Paul, 2008). De ce point de vue, envisager les formes d'accumulation et de différenciation au sein d'une société paysanne suppose d'analyser, non seulement les modalités de la production agricole, mais également celles de la (re) production des producteurs. L'articulation des rapports sociaux de production et de reproduction définit un « modèle socioéconomique » (Meillassoux, 1977) ou « un système social »(Paul, 2003). La reconstitution de ce modèle a valeur heuristique : on caractérise le fonctionnement d'un système social historique précisément pour expliquer sa crise.

Le concept de système social a une portée très générale en anthropologie économique. Le modèle de la « communauté domestique » chez Meillassoux s'applique à toutes les sociétés paysannes dont le fonctionnement repose sur la circulation du produit entre les générations et la mobilité des capacités génésiques entre les cellules productives (Paul, 2008). À l'échelle d'une petite région, une situation agraire est une réalité complexe qu'il s'agit de saisir dans toutes ses dimensions : écologiques, techniques, sociales et économiques. Le concept de système agraire, appliqué à cette échelle, intègre ces différentes dimensions (Cochet, 2011) et englobe le concept de système social. Cette approche permet de penser le « technique » et l' « écologique » avec l' « économique » et le « social », et surtout d'identifier, au niveau des interactions entre ces éléments de différentes natures, ce qui permet au système de se reproduire à l'identique, de se transformer, de se développer ou, au contraire, de rentrer en crise.

L'analyse des disparités et des processus de différenciation au sein de la paysannerie repose dans cette étude sur le calcul économique du revenu à l'échelle des unités de production/consommation. Cette évaluation des performances techniques et économiques découle de l'analyse historique. En effet, le partage de la valeur ajoutée et la formation du revenu dépendent des modalités d'accès aux ressources productives qui résultent de l'évolution historique des rapports sociaux.

L'approche historique a guidé la méthodologie de notre enquête. Quelle que soit la qualité des données collectées, la validité des résultats repose, en premier lieu, sur l'échantillonnage des unités de production/ consommation (Couty, 1983 ; Passeron, 2006). Dans cette étude, l'échantillonnage a reposé sur un zonage agro-écologique ${ }^{5}$

5. La description de ce zonage ne peut être détaillée dans le cadre de cet article. Il fait l'objet d'une analyse spécifique dans Palliere (2014). 
et sur l'analyse historique de la trajectoire des unités de production/consommation. Cette dernière révèle l'importance de la marchandisation de la force de travail dans les processus de différenciation au sein de la paysannerie. Nous sommes donc partis d'une pré-typologie simple : capteurs et pourvoyeurs de journées de travail. Au fur et à mesure des enquêtes, nous avons affiné cette pré-typologie pour finalement la dépasser en évaluant plus précisément les ressources engagées par chaque unité de production/consommation pour la captation de force de travail.

En pratique, l'analyse historique repose essentiellement sur les récits de vie des habitants des villages de Sella Limba collectés à l'occasion de plusieurs séjours sur le terrain entre 2007 et 2011. Des entretiens individuels (304) ont été réalisés avec des paysans de différentes générations dans 75 villages différents. Pour $27 \%$ d'entre eux avec des femmes. Trois sujets étaient notamment abordés lors de ces entretiens : l'évolution des paysages cultivés, des pratiques agricoles et des rapports sociaux de production et de consommation. Des sources écrites (récits de voyageurs, archives coloniales, photos aériennes, cartes) ont également été mobilisées, notamment pour étudier les héritages historiques plus anciens ou pour recouper les témoignages oraux.

Les calculs des performances techniques et économiques à l'échelle des unités de production/consommation reposent sur 58 études de cas, réalisées dans 21 villages différents. Les enquêtes commençaient toujours avec l'homme désigné comme le « chef de la maison ». Ce terme ne doit pas tromper : comme dans de nombreuses situations africaines (Gastellu, 1980), l'unité de résidence ne correspond que rarement aux contours des unités de production/consommation. Définir sa composition (actifs et consommateurs) était donc notre premier objectif. Certaines activités sont conduites sous la responsabilité du chef, mais d'autres sont individuelles. Pour avoir une vue d'ensemble des activités agricoles et extra-agricoles, il était donc indispensable, pour chaque cas d'étude, d'interroger individuellement tous les membres actifs de l'unité de production/consommation. La trajectoire de chacun était reconstituée : comment a-til rejoint la «maison », depuis combien d'années travaille-t-il ? La contribution en travail de chacun était précisée, notamment en comptabilisant les journées de travail effectuées, achetées ou cédées à différents moments au cours de l'année écoulée pour différentes activités (agricoles ou extra-agricoles, individuelles ou collectives). L'institution des groupes de travail saisonniers, à laquelle participent presque tous les hommes et les femmes en âge de travailler, facilite grandement la collecte de ces informations. Les femmes adultes étaient en plus interrogées sur les calendriers d'alimentation. Ce dispositif d'enquêtes relativement lourd supposait plusieurs visites, espacées de plusieurs mois, voire de plusieurs années, ce qui permettait également de considérer l'évolution à court terme de ces unités.

La Sella Limba est un chiefdom de 400 $\mathrm{km}^{2}$ au nord de la Sierra Leone (figure 1). Le climat est tropical humide, avec une saison des pluies (1 $800 \mathrm{~mm}$ de précipitations annuelles) et une saison sèche bien marquées. On distingue cinq grandes zones agro-écologiques dans la région étudiée. La plaine fluviale de l'ouest est caractérisée par la présence de cuvettes inondées (bolilands). Au centre, le relief est composé de collines aux formes allongées séparées par des bas-fonds. Les friches arborées qui dominent au nord sont remplacées par une savane herbeuse vers le sud. Enfin, à l'est, le relief devient plus escarpé et le paysage est une mosaïque complexe de savanes arborées et de friches arborées. D'après le dernier recensement (Statistics Sierra Leone, 
2006), la densité rurale du chiefdom est de $106 \mathrm{hab} / \mathrm{km}^{2}$ (contre une moyenne nationale de $70 \mathrm{hab} / \mathrm{km}^{2}$ ). Mais le peuplement est hétérogène : les marges est et ouest, dominées par la savane arborée sont faiblement peuplées (entre 30 et $40 \mathrm{hab} / \mathrm{km}^{2}$ ) et le centre du chiefdom concentre $80 \%$ de la population rurale (d'après nos propres comptages). Partout, la société paysanne s'organise en petits villages (en moyenne 284 habitants et 30 unités de production/ consommation par village) avec chacun un territoire bien défini.

La diversité des paysages traduit celle des modes d'exploitation du milieu (Palliere, 2014). L'abattis-brûlis et l'arrachage des savanes arborées, qui reposent sur de longues périodes de friches, s'opposent à la pratique du billonnage des savanes herbeuses. La riziculture inondée dans les bas-fonds a plus ou moins d'importance d'une zone à l'autre de la région étudiée. Tous les producteurs combinent cultures vivrières essentiellement autoconsommées (céréales, huile de palme) et cultures commerciales (notamment piment). À l'échelle de la région étudiée, on observe une grande diversité de pratiques mais, dans une zone agro-écologique donnée, aucune spécialisation des « systèmes de production ».

\section{Intégration aux marchés et transformation des rapports sociaux au $X X^{\mathrm{e}}$ siècle}

\section{Fonctionnement des grandes unités de production/consommation (première moitié du $X X^{\mathrm{e}}$ siècle)}

Les plus âgés des paysans de Sella Limba ont aujourd'hui entre 80 et 90 ans. On peut estimer que leurs plus anciens souvenirs remontent aux années 1930-1940. Avec plus de $30 \mathrm{hab} / \mathrm{km}^{2}$, la Sella Limba était déjà une zone densément peuplée au regard du Nord de la Sierra Leone (McCulloch, 1950). Le paysage était dominé par les friches arborées et l'abattis-brûlis était le principal mode d'exploitation du milieu. Chaque parcelle était exploitée deux années de suite avant d'être abandonnée 5 à 10 ans. Ces informations sont confirmées par : la description de l'outillage (hache, sabre d'abatis et houe à fer étroit et courbe) ; le nom des variétés de riz utilisées (la variété disi, aujourd'hui disparue de la région, décrite comme particulièrement adaptée à la riziculture d'abatis-brûlis sur les recrûs arborés) et les récits des voyageurs de la première moitié du $\mathrm{XX}^{\mathrm{e}}$ siècle (Migeod, 1926). Les bas-fonds, inondés en saison des pluies, n'étaient alors pas exploités en tant que tels.

Seule une part marginale du produit agricole faisait l'objet d'échanges marchands. La Sella Limba était relativement isolée par rapport aux routes commerciales de l'époque coloniale : comptoirs commerciaux à l'embouchure des fleuves, stations du chemin de fer (Riddell, 1970 ; Deveneaux, 1973). Les revenus monétaires tirés des palmistes permettaient essentiellement de s'acquitter de l'impôt colonial, la hut tax mise en place en même temps que le protectorat britannique en 1896. Les jeunes hommes ou les jeunes femmes de cette génération n'avaient aucune possibilité de vendre leur force de travail contre salaire, même hors du chiefdom.

Du point de vue de l'organisation sociale de la production, tous les témoins de l'époque décrivent des unités de production/consommation rassemblant un grand nombre d'actifs placés sous l'autorité d'un homme âgé, l'aîné, et travaillant tous dans un grand essart. Après la récolte, le paddy était placé dans un coffre en bois sous le contrôle de l'aîné. Il était responsable de la conservation des semences et de la distribution quotidienne du paddy à ses épouses.

Vers 1930, dans le village de Kamawanka par exemple, deux unités de production/ consommation rassemblaient chacune une cinquantaine de personnes et une vingtaine 
d'actifs. L'évocation d'unités de grande taille durant la première moitié du $\mathrm{XX}^{\mathrm{e}}$ siècle est fréquente dans la littérature (Little, 1948 ; Richards et al., 2004). Cette situation suppose une subordination stricte du travail des cadet(te)s par les aîné(e)s. Elle reposait sur le contrôle des semences, des outils et du foncier, mais également des rapports matrimoniaux. Dans cette société virilocale et patrilinéaire, la circulation des femmes entre les villages était en effet le monopole des aînés et de leurs épouses. Les témoins de l'époque insistent sur l'âge tardif auquel les hommes se mariaient et, à l'inverse, le jeune âge auquel les femmes étaient placées chez leur future belle-mère.

On observait un réajustement permanent de l'équilibre entre les capacités productives et le nombre de bouches à nourrir. Ce réajustement prenait la forme d'une circulation enfantine intense entre épouses au sein des unités de production/consommation et entre unités au sein des aires matrimoniales. L'importance de la circulation enfantine repose sur la pluralité des droits sur la progéniture d'une femme (Bledsoe et Isiugo-Abanihe, 1989) : un enfant récemment né était élevé soit par sa grand-mère paternelle, dans le village de son père, soit par sa grand-mère maternelle, dans le village de son oncle utérin, généralement situé à quelques kilomètres. Les enfants gardaient néanmoins le nom de « clan » de leur patrilignage et la mémoire de leur origine. C'est ce qui explique la coexistence, dans tous les villages de Sella Limba, de nombreux patrilignages différents, les uns se disant les oncles et les autres neveux.

La distinction que fait Meillassoux (1975) entre énergie et force de travail paraît ici pertinente. Au sein de ces grandes unités de production/consommation, l'énergie de travail ne trouvait emploi que dans le cycle viager au cours duquel elle était convertie en subsistances, redistribuées aux futurs, actuels et anciens producteurs. Elle n'existait pas sous sa forme moderne de force de travail, c'est-à-dire du travail en quantités discrètes, autonomisées du reste de la vie sociale. Symétriquement, le produit agricole se présentait essentiellement sous forme de subsistance et presque jamais de marchandise. À un moment « $\mathrm{t}$ », le statut des individus, en fonction de leur position dans le cycle viager, pouvait être inégal. Ainsi, alors que de nombreux hommes décédaient sans progéniture socialement reconnue, les aînés étaient souvent mariés à plus de 3 ou 4 épouses. Le fonctionnement quasi autarcique des unités de production/ consommation ne laissait, par contre, que très peu de place à la différenciation économique ou sociale entre elles.

\section{Diamants alluviaux et riziculture inondée}

\section{L'éclatement des grandes unités} de production/consommation (1956-1973) Dans les années 1950, ces rapports sociaux sont ébranlés par l'intégration économique de la région. L'exploitation des diamants alluviaux dans les districts de l'est connaît alors un essor spectaculaire (Zack-Williams, 1982). Ne requérant que très peu de capital et beaucoup de maind'œuvre, le secteur attira de nombreux jeunes paysans de tout le pays (Swindell, 1974). La croissance de la population non agricole suscita le développement d'un marché vivrier national (Binns, 1982). En parallèle, les infrastructures de transport se développèrent rapidement (Riddell, 1970). Ainsi, des commerçants, libanais ou kryo, s'installèrent en Sella Limba à la recherche de riz à exporter vers les districts diamantifères.

Dans le secteur diamantifère, la force de travail comme les subsistances se présentaient comme des marchandises. L'émergence de rapports sociaux marchands, même loin du village, ne pouvait laisser indemne le fonctionnement des grandes unités de production/consommation. Du point de vue des aînés, qui contrôlaient 


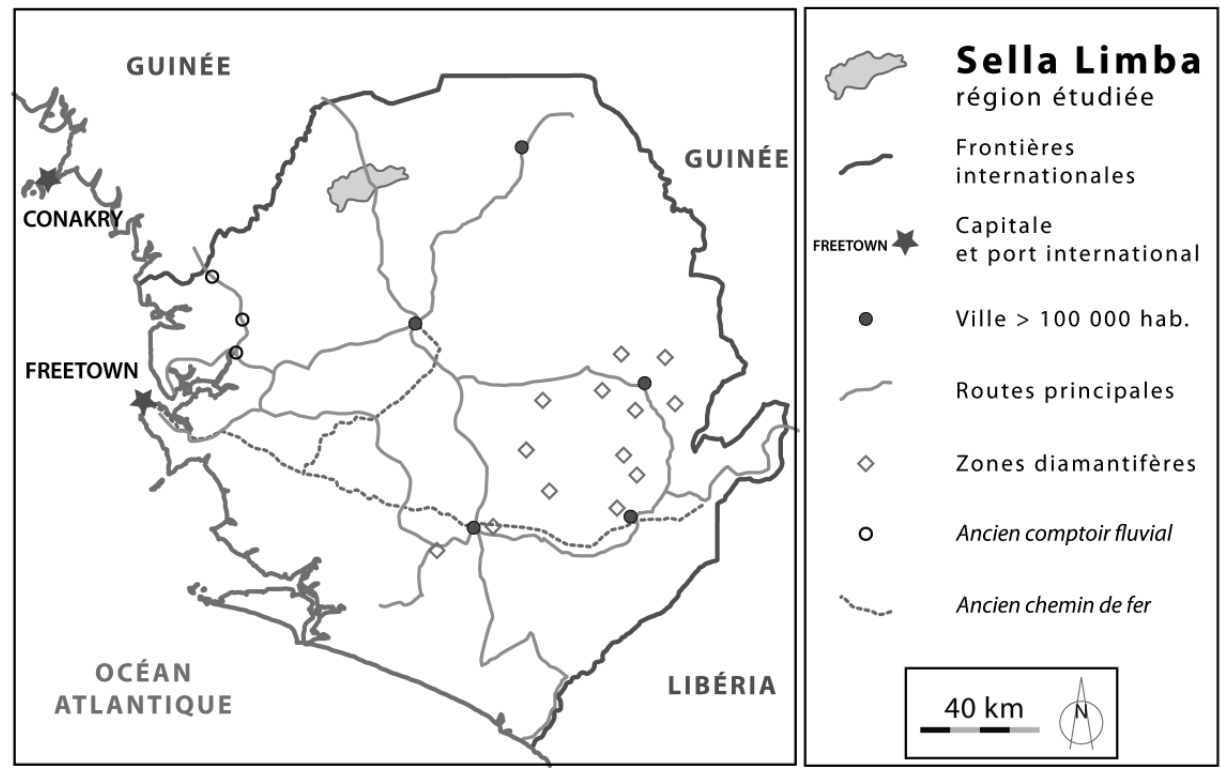

Source : I'auteur, fond de carte : histgeo.ac-aix-marseille.fr.

le gros des récoltes, le développement d'un marché vivrier offrait la possibilité d'accéder à de nouveaux biens de consommation : tôles ondulées pour le toit des maisons, postes radio, vêtements, etc. Du point de vue des cadets, partir et vendre sa force de travail dans les districts diamantifères représentait une opportunité inédite d'émancipation. Nombre d'entre eux l'ont d'ailleurs saisie. Cependant, les aînés ne pouvaient accepter d'être privés de cette énergie de travail à la formation de laquelle ils avaient contribué. On retrouve dans les témoignages ces tensions domestiques dont une des manifestations est la multiplication des actes de sorcelleries, généralement dirigés contre les cadets qui quittaient le village « sans dire au revoir» et manquaient de « respect » à leurs aînés.

En Sella Limba, la riziculture inondée dans les bas-fonds se développe à cette même époque. Des pratiques plus exigeantes en travail, défriche, labour et repiquage, sont massivement adoptées pour permettre une exploitation plus régulière des parcelles : quatre années de culture consécutives pour deux années de recrû. L'acquisition d'outils spécifiques et de nouvelles variétés de riz sont les innovations qui marquent dans les témoignages cette transformation des modes d'exploitation du milieu.

Avec moins de $50 \mathrm{hab} / \mathrm{km}^{2}$ en 1950 (Clarke, 1969), l'épuisement des terres pluviales ne semble pas avoir été le facteur déterminant de ce processus d' " intensification par le travail » selon la définition de Jouve (2006). Les acteurs de ces innovations, aujourd'hui des paysans âgés, insistent plutôt sur la volonté de profiter des nouvelles opportunités offertes par l'émergence du marché vivrier. En adoptant la riziculture inondée, ils n'ont pas abandonné la culture pluviale d'abatis-brûlis. Son intégration dans les systèmes de production a permis un remplissage des calendriers de travail traditionnels, les bas-fonds étant repiqués après l'implantation du riz dans 
les parcelles pluviales (Palliere, 2013). En augmentant la surface cultivée, les producteurs étaient en mesure de dégager un surplus commercialisable plus important.

Le développement de la riziculture inondée a offert l'occasion de résoudre une partie des tensions domestiques. En échange du travail fourni, l'aîné attribuait aux cadets une parcelle en bas-fond. Après les semailles dans la "grande parcelle » du vieux, les jeunes hommes descendaient dans les zones basses labourer et repiquer leurs « petites parcelles ». Les récoltes issues de ces dernières ne remontaient pas jusqu'aux coffres de l'aîné. La possibilité, pour les cadets, de jouir d'une partie de leur force de travail s'accompagne de leur émancipation progressive au village. Mais, les bas-fonds des cadets représentaient de petites surfaces, 10 à 20 ares $(10$ à $20 \mathrm{~kg}$ de paddy semés en pépinière). L'essentiel du travail et du produit restait sous le contrôle de l'aîné. À sa mort, néanmoins, l'unité production/consommation pouvait se réorganiser autour des groupes de frères utérins qui refusaient de se placer sous l'autorité de leur oncle paternel. $\mathrm{S}$ 'amorce dès cette époque la tendance à l'éclatement des grandes unités de production/consommation. Il faut cette tendance de la segmentation lignagère qui, tout en résolvant les tensions domestiques, assurait la reproduction à l'identique et l'extension spatiale du système social. Avec leur éclatement, au contraire, les unités de production/consommation de taille toujours plus réduite ne peuvent plus fonctionner en autarcie comme naguère.

\section{De l'agriculture contractuelle à la crise agraire actuelle (1978 - aujourd'hui)}

Le marché vivrier national a été sacrifié par les importations massives de riz à bas prix dès le début des années 1970 (Reno, 1995 ; Richards, 1996). À l'échelle nationale, après une phase de croissance dans les années 1950-1960, la production de riz stagne, puis décline (Phelinas, 1986). C'est dans ce contexte qu'en 1973, une filière contractuelle de production de tabac est mise en place par la British and American Tobacco.

La compagnie avançait les frais de campagne, notamment les engrais azotés et les tabaculteurs sous contrat s'engageaient à suivre un strict cahier des charges. L'itinéraire technique obligatoire était très lourd et rentrait en concurrence avec celui des cultures vivrières, notamment pluviales : la défriche brûlis, le dessouchage des recrûs arborés et le billonnage des parcelles avaient lieu pendant la préparation des essarts. La récolte, le traitement et le conditionnement des feuilles monopolisaient un actif complet au cœur de la saison des pluies entre mai et juillet, soit pendant la fenêtre calendaire de l'implantation du riz pluvial. Pour inciter l'abandon de la culture du riz d'abattis brûlis et favoriser la tabaculture, la compagnie proposait aux producteurs sous contrat des sacs de riz importé, payables à la récolte. Jusqu'à cette époque, la part du produit agricole domestique vendue sur le marché restait faible et était destinée à l'achat de produits manufacturés « de luxe». Au sein de la filière intégrée, un nombre croissant d'unités de production/consommation vendait une part importante du produit de leur travail pour se procurer leur subsistance. Ainsi, dans une région voisine, Binder (1989) note que $75 \%$ du revenu monétaire des tabaculteurs était mobilisé pour le remboursement ou l'achat de riz importé fourni par la compagnie. Cette monétarisation du revenu agricole implique un rapprochement entre la journée de travail agricole et sa valeur monétaire. Les tensions entre l'aîné, qui maitrisait encore le plus gros des revenus, et les cadets, qui travaillaient dans les parcelles, se sont exacerbées.

Une amorce de différenciation au sein de la paysannerie a vu le jour à l'époque de la tabaculture sous contrat. Lors des 
premières campagnes, presque tous les hommes adultes à la tête d'une unité de production/consommation ont signé des contrats avec la compagnie. Chaque producteur recevait un ou deux sacs d'engrais de $25 \mathrm{~kg}$ (granulés), correspondant à des parcelles de 10 à 20 ares. Les unités de production/consommation qui rassemblaient plus d'actifs pouvaient mieux faire face à la diversité des travaux agricoles qu'exigeait la conduite en parallèle d'une parcelle de tabac sous contrat et des parcelles vivrières. Souvent, sous la direction de l'aîné, un travailleur adulte était responsable de la tabaculture et un autre de la production vivrière domestique. L'intégration dans la filière a été sans conteste une source d'enrichissement pour ces gros tabaculteurs. Les revenus dégagés leur ont permis de faire grossir leurs troupeaux de petits ruminants, construire des maisons et, plus important, d'envoyer des enfants faire des études en ville. Mais ils devaient partager, d'une manière ou d'une autre, une partie des revenus engrangés. Ainsi, de nombreux enfants en âge de travailler étaient placés chez ces paysans prospères qui leur offraient la possibilité d'aller à l'école en échange de leur participation aux travaux des champs. Mais s'ils n'étaient plus en mesure de satisfaire les aspirations des hommes et des femmes, placés sous leur autorité, la cohésion des unités de production/consommation était menacée.

Pour les plus petites unités, il était beaucoup plus difficile de jouer la carte de la diversification vivrière et commerciale. Plus fragiles structurellement, elles étaient sujettes à des déficits en capital circulant. Pour obtenir par exemple les semences dont ils manquaient en début de cycle, certains individus ont commencé à cette époque à échanger des journées de travail contre du paddy ou de l'argent. Alors que, dans les années 1950-1960, vendre sa force de travail n'était possible que dans les districts diamantifères, l'éclatement des groupes domestiques conjugué à la monétarisation croissante du revenu des producteurs expliquent l'émergence, au sein des villages, d'échanges marchands de force de travail contre du riz ou de l'argent.

En 1996, l'extension au nord du pays de la guerre civile, démarrée en 1991 à la frontière libérienne, met fin aux opérations de la compagnie de tabac. La guerre civile est d'abord le produit de la chute d'un régime clientéliste entraînant avec lui la société sierra-léonaise tout entière (Zack-Williams, 1999 ; Richards, 1996). Le Revolutionary United Front (RUF) a principalement recruté dans les districts diamantifères parmi la « jeunesse hyper-mobile », frange marginalisée de la population d'origine rurale (Chauveau et Richards, 2008). En Sella Limba, les exactions et extorsions du RUF ont entraîné la décapitalisation brutale des paysans les plus prospères, avec notamment le racket des petits ruminants dans les villages.

Cette crise politique, économique et sociale à l'échelle nationale entre en résonance avec une crise agraire locale. Alors que la densité de population avait beaucoup augmenté, l'arrachage des recrûs arborés pour la culture du tabac a entrainé le recul brutal des recrûs arborés au profit d'une savane herbeuse traversée chaque année par le feu (Palliere, 2013). Depuis 1996, les paysans n'ont plus accès aux engrais de synthèse. Pour faire face à la dégradation de la fertilité, un nouveau mode d'exploitation du milieu a été adopté : les cultures sur billon (manioc ou piment) alternent avec les céréales cultivées à « plat » (riz à cycle court ou fonio) et une association de légumineuse (arachide et pois cajan). Le poids du désherbage dans les calendriers de travail a considérablement augmenté. La riziculture dans les bas-fonds s'est intensifiée, les parcelles n'étant abandonnées qu'environ une année sur six. Dans ce système, l'essentiel de la charge de travail est concentré entre mai et août, période durant laquelle 
la préparation des parcelles (billonnage, labour), l'implantation des cultures (semis, repiquage) et l'entretien des parcelles (désherbage) doivent être réalisés.

Sans accès, depuis la fin des opérations de la compagnie de tabac, à des moyens de production plus performants, c'est la diversification des activités agricoles et extraagricoles qui permet de compenser la baisse de la productivité journalière résultant de l'intensification en travail des modes d'exploitation du milieu. La multiplication des petites parcelles, la mise à profit de toutes les hétérogénéités du milieu cultivé, l'usage raisonné de la diversité des cultures et des variétés permettent d'étaler au mieux les travaux agricoles durant toute la saison des pluies. Les femmes profitent de la contresaison pour transformer certains produits agricoles périssables en denrées stockables et commercialisables (huile de palme et farine de manioc). Les jeunes hommes migrent temporairement à Freetown et, depuis l'explosion des cours mondiaux de l'or en 2007, travaillent dans les sites d'orpaillage situés dans la région même.

La tendance à la monétarisation du revenu des producteurs s'est accélérée pendant l'époque de la tabaculture contractuelle. Depuis la guerre civile, le piment destiné aux marchés urbains est devenu la culture commerciale principale, assurant le gros du revenu monétaire. D'après nos enquêtes, ce dernier représente aujourd'hui $40 \%$ du revenu agricole et le riz importé environ la moitié des céréales consommées dans les villages. Par ailleurs, la tendance à l'éclatement en unités de production/consommation, amorcée dès les années 1950, s'est poursuivie de génération en génération. Le village de Kamawanka, que nous évoquons plus haut, rassemblait (en 2011) 244 personnes et 35 unités de production/ consommation, en moyenne 7 personnes et 3 actifs/unité.

Conjuguées, ces deux tendances sont à l'origine de l'émergence d'échanges marchands de journées de travail entre les unités de production/consommation. La grande majorité des échanges de journées de travail se fait via l'institution des groupes de travail saisonniers ${ }^{6}$. La contribution de chaque membre est soumise à une comptabilité rigoureuse : pour un groupe de 20 membres, par exemple, chacun disposera 5 fois des 20 travailleurs entre mai et août. On peut vendre son tour du groupe en échange de riz ou d'argent. Cela arrive notamment quand un paysan manque de semences, doit payer des soins pour un enfant, ou simplement n'a plus de riz dans le grenier pour nourrir sa maison pendant la soudure. Cette situation, inimaginable à l'époque des grandes unités de production/ consommation, est aujourd'hui courante. Un jour de travail dans ces échanges équivaut à un pan $(2,5 \mathrm{~kg})$ de paddy. Ainsi, au cœur de la saison des pluies, alors que chacun se consacre à lutter contre l'enherbement de ses parcelles, la vente d'un tour de son groupe de 20 membres permet à une femme de nourrir 5 personnes pendant une dizaine de jours.

La monétarisation du foncier s'est largement développée dans les dernières décennies en Afrique de l'Ouest (Chauveau et al., 2006). En Sella Limba pourtant, la marchandisation de la force de travail en Sella Limba ne s'est pas accompagnée de celle de la terre, y compris dans les zones agro-écologiques où la densité de population dépasse $100 \mathrm{hab} / \mathrm{km}^{2}$. Le foncier ne constitue donc pas une opportunité de capitalisation. Dans cette région qui se caractérise au contraire par un solde migratoire négatif depuis les années 1950, la notion d' « étranger » ne prend pas dans les rapports fonciers le sens qu'on lui connaît par exemple en Côte d'Ivoire (voir notamment Chauveau, 2010). Dans chaque village, les aînés des deux ou trois patrilignages concentrent les droits d'attribution des

6. Organisation du travail courante en Afrique subsaharienne (Richards, 1983). 
droits d'usage. Dans ces territoires maillés de petites communautés entretenant entre elles des liens matrimoniaux étroits, tous les producteurs d'un village donné ont, par filiation ou par alliance, des liens avec les patrilignages fondateurs. L'éclatement des groupes domestiques a certes entraîné la multiplication des détenteurs de droits d'usage et le développement de nouvelles pratiques foncières, comme le transfert d'une part symbolique de la récolte à l'aîné en fin de cycle. Mais l'enjeu du foncier reste subordonné à celui de la captation de la force de travail.

\section{Circulation de la force de travail et des enfants}

Nous avons identifié la marchandisation des rapports sociaux et notamment des échanges de force de travail au sein des villages comme une tendance essentielle de la trajectoire historique du système agraire. Alors que tous les producteurs ont accès au même outillage et pratiquent globalement les mêmes combinaisons de cultures, en captant de la force de travail au moment où elle atteint son coût d'opportunité maximum, entre mai et août, certains producteurs sont en mesure d'augmenter la surface qu'ils cultivent une année donnée au détriment des pourvoyeurs de ces journées de travail. Alors que l'on n'observe aucune accumulation dans des moyens de production ou dans la ressource foncière, ces échanges marchands de force de travail sont-ils à l'origine d'une différenciation durable de la paysannerie ? Pour répondre à cette question, nous allons chercher à évaluer le revenu économique des unités de production/consommation en fonction de leur capacité à capter de la force de travail.

\section{Circulation marchande de la force de travail et disparités économiques}

L'estimation du Revenu agricole (RA) suppose d'évaluer la production agricole physique et sa valeur monétaire à l'échelle des parcelles puis de l'unité de production/consommation dans son ensemble. La collecte des données agro-économiques repose, de manière générale, sur l'identification d'un nombre limité de systèmes de culture $^{7}$ et de production notamment grâce à l'analyse du paysage de la région étudiée (Palliere, 2014). Plus spécifiquement, pour la mesure des rendements, nous avons opté pour une méthode combinant mesures directes et mesures indirectes de la production. La méthode des carrés de rendements s'est révélée inadaptée tant à nos objectifs qu'aux conditions locales de culture du fait notamment de l'hétérogénéité des parcelles (Milleville 1972). Les mesures directes étaient donc réalisées à l'échelle des parcelles entières. Compter le nombre de travailleurs nécessaires pour la récolte, notamment, permet une approximation de la récolte (Delarue, 2007). Les mesures indirectes consistaient en des entretiens au sujet de la répartition du produit entre autoconsommation, vente et dons à différents stades de la récolte. Ces mesures ont permis de faire pour chaque culture, en association ou en rotation dans les parcelles en Sella Limba, une hypothèse de rendement moyen.

La valeur monétaire des cultures commercialisées pour une grande part (piment et arachide essentiellement) est évaluée au prix où le producteur nous informe les avoir vendues ou, en l'absence de cette information, au prix au moment de la récolte, c'est-à-dire quand la majorité des producteurs vendent le gros de leur production. La valeur des cultures essentiellement autoconsommées est estimée à leur coût d'opportunité : les céréales au prix moyen

7. «L'ensemble des modalités techniques mises en œuvre sur des parcelles traitées de manière identique. Chaque système de culture se définit par (i) la nature des cultures et leur ordre de succession ; (ii) les itinéraires techniques appliqués à ces différentes cultures, ce qui inclut le choix des variétés pour les cultures retenues » (Sébillotte, 1977). 
Figure 2. Économie des processus productifs : définition et calcul des grandeurs économiques

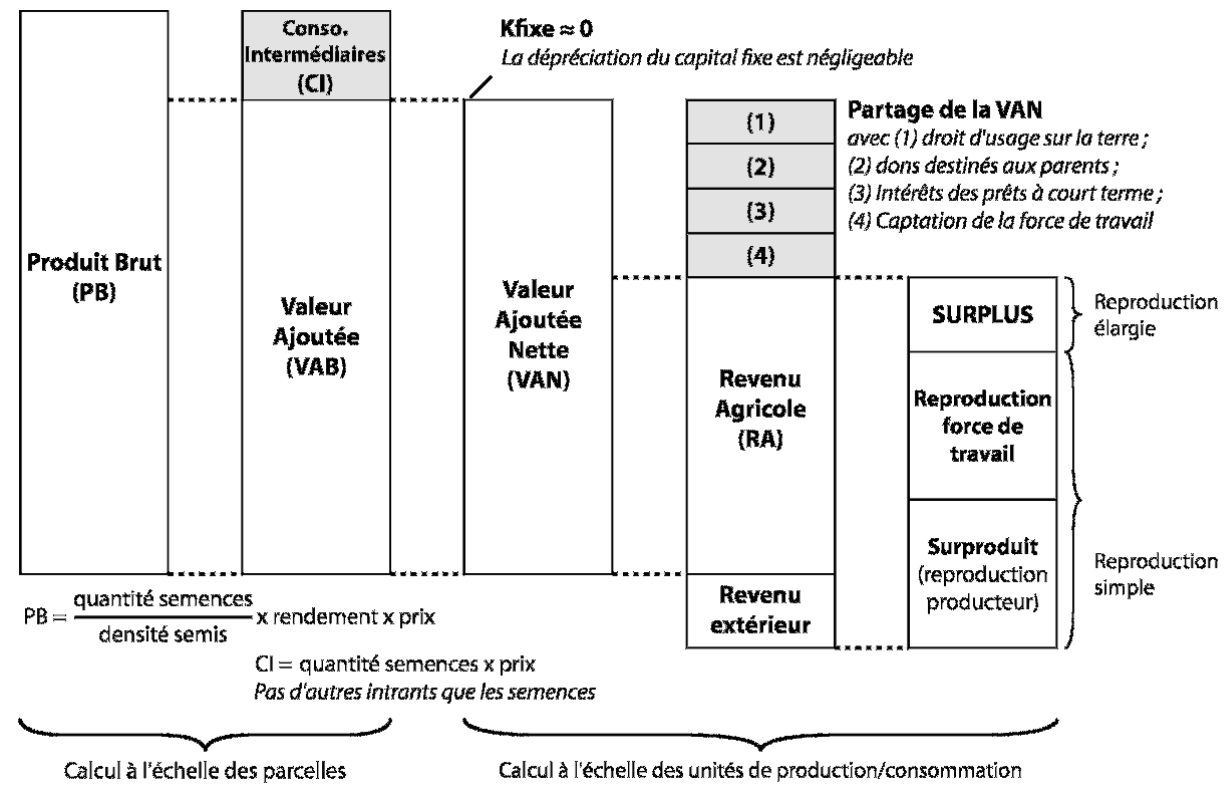

Source : I'auteur adapté de Cochet (2011)

sur l'année du riz importé, consommé massivement par les paysans en période de soudure, et les régimes de palmiers à leur prix au village non transformés. Ces hypothèses de rendements et de prix sont utilisées pour calculer la Valeur ajoutée nette (VAN). Pour chaque cas d'étude, nous avons collecté la quantité de semences utilisée dans chaque parcelle. De cette information on déduit les surfaces cultivées, la production et sa valeur monétaire.

Pour déterminer le RA, il faut déduire de la VAN : la part, fixe, des récoltes qui revient aux aînés octroyant le droit d'usage de la terre ; les dons, très variables, destinés notamment aux parents en ville ; éventuellement la part qui revient aux commerçants locaux comme intérêts des prêts à court terme concédés pendant la soudure ; enfin, et nous serons particulièrement attentif à cette modalité de partage de la VAN, les ressources, en argent ou en nature, engagées pour capter de la force de travail extérieure (figure 2).
En effet, on détermine ensuite les ressources engagées par chaque unité de production/consommation pour la captation de force de travail extra-domestique en fonction du nombre de journées captées ou cédées par les différents membres actifs. Pendant le pic de travail entre mai et août, la journée de travail agricole coûte $0,9 € /$ travailleur/jour ${ }^{8}$ (en nature ou en argent), ce prix étant remarquablement stable d'un village à l'autre en Sella Limba. Il faut y ajouter le coût de la préparation du repas qu'il faut offrir aux travailleurs à la pause (0,4 €/travailleur). À partir de septembre, les travailleurs sont « remerciés » avec une part de la récolte. Les ressources engagées sont alors proportionnelles aux surfaces mises en culture et ne proviennent plus d'un capital circulant accumulé durant les cycles de culture précédents. En moyenne

8. Pour faciliter la lecture du texte, tous les prix sont indiqués en euros de l'année 2011 ( 1 € 2011 5500 leones 2011). 
les unités de production/consommation engagent $35 € /$ an pour la captation de force de travail extérieure entre mai et août. Les mieux dotées en capital circulant consacrent jusqu'à plus de 250 €/an. À l'opposé, les unités «pourvoyeuses » reçoivent des ressources (argent ou semences) contre les journées de travail cédées, et ce jusqu'à l'équivalent de $65 € / a n$.

Pour comparer les cas d'études entre eux, on ramène le revenu agricole comme les ressources consacrées à la captation de force de travail entre mai et août, au nombre d'actifs domestiques (figure 3). Les résultats donnent une idée des disparités économiques qui résultent des échanges de force de travail entre les groupes domestiques. Sans surprise, les unités de production/ consommation qui consacrent des ressources importantes pour capter de la force de travail entre mai et août (plus de $18 €$ / an/actif domestique) dégagent presque tous des RA importants (plus de $360 € / \mathrm{an} /$ actif domestique). Inversement, celles qui cèdent des journées de travail dégagent souvent des RA inférieurs à $182 € /$ an/actif domestique. On observe cependant, pour un même niveau de ressources consacrées à la captation de force de travail, des différences importantes de RA. Ces écarts sont le produit des différentes combinaisons de cultures pratiquées une année donnée, notamment en fonction des conditions agro-écologiques dans lesquelles sont placés les paysans, et des prix inégaux auxquels ils vendent leurs productions.

\section{Circulation enfantine et absence de différenciation économique entre unités de production/consommation}

En Sella Limba, une part importante du revenu des unités de production/consommation est dévolue à leur reproduction simple. On peut distinguer théoriquement la part nécessaire à la reproduction de la force de travail à court terme, c'est-àdire l'entretien des producteurs actuels, et le surproduit autorisant la « reproduction des producteurs » (Meillassoux 1975), c'est-à-dire la formation des futurs actifs et l'entretien des anciens. Nous avons estimé, dans les conditions économiques et sociales de la région étudiée, à $150 € /$ an/consommateur ${ }^{9}$ le seuil de reproduction simple. Il s'agit essentiellement de la valeur de la consommation alimentaire $^{10}$. Le coût des autres besoins vitaux (habillement, hygiène) est négligeable par rapport à celui de l'alimentation. Les soins médicaux ne peuvent être considérés dans le seuil de « survie », l'ensemble des paysans n'y ayant pas accès.

Cette part, qui dépend du nombre de consommateurs dans chaque unité doit être retranchée au revenu pour estimer le surplus, définit comme la part du revenu disponible pour la reproduction élargie des unités de production/consommation, c'est-à-dire, par opposition à la reproduction simple, pour l'accumulation dans des moyens de production plus performants ou pour la couverture de besoins qui ne relèvent pas du simple entretien des producteurs. La comparaison d'unités de production/consommation de tailles différentes nécessite de ramener le surplus estimé au nombre d'actifs domestiques.

À ce stade, nous prenons en compte les activités extra-agricoles. Même si les salaires journaliers sont très faibles $(1,8 € /$ jour

9. On distingue le nombre de consommateurs du nombre de «bouches à nourrir ». Le second est pondéré par l'âge des individus pour obtenir le premier : on compte 1 consommateur par adulte du groupe et 0,5 consommateur par enfant, c'està-dire un individu trop jeune pour participer à un groupe de travail (kune). Cette pondération grossière est inspirée de la manière dont les cuisinières calculent la ration en riz pour leur marmite ( 1 « cup » de riz décortiqué par adulte et 0,5 « cup » par enfant).

10. Pour un adulte : $126 \mathrm{~kg}$ de riz blanc, $14 \mathrm{~kg}$ d'huile de palme, $18 \mathrm{~kg}$ d'arachide coque, $90 \mathrm{~kg}$ de manioc frais, $6 \mathrm{~kg}$ de pois cajan et l'équivalent de 41,30 € d'ingrédients achetés (poissons, glutamate, sel...). 
en moyenne) et inférieurs à la productivité journalière du travail agricole (Palliere, 2014), la vente de journées de travail dans les mines d'or artisanales de la région offre depuis 2008 l'opportunité aux jeunes hommes d'engranger un revenu en toute indépendance et de ne pas peser sur le budget domestique pendant les périodes de creux du calendrier de travail agricole. Dans l'hypothèse la plus favorable, un actif agricole peut travailler dans les sites d'orpaillage 5 mois dans l'année et sa rémunération totale pourra atteindre au maximum $180 €$ dont la majeure partie sera consommée sur place pour le transport, la nourriture et le logement. Dans les revenus extra-agricoles, il faut aussi considérer le produit de la vente des journées de travail pour les unités pourvoyeuses de force de travail. Ramenés à l'échelle des unités de production/consommation, les revenus extra-agricoles représentent en moyenne $10 \%$ des revenus totaux et $22 \%$ si l'on considère uniquement les unités avec au moins un jeune pratiquant l'orpaillage.

Le niveau des surplus est globalement faible. Quelques unités de production/ consommation dégagent un surplus significatif, plus de $130 € /$ an/actif domestique. Mais plus nombreuses sont celles pour lesquelles il est faible, inférieur à $40 € / a n /$ actif domestique.

Il faut comparer ces niveaux de surplus à la valeur des biens matériels auxquels les paysans aspirent aujourd'hui dans les campagnes sierra-léonaises. Mis à part quelques ustensiles domestiques en plastique ou en émail, les foyers sont très pauvrement équipés. Si le téléphone portable (environ $9 €$ ) s'est démocratisé dans les campagnes ces dernières années, les frais médicaux « modernes » restent inaccessibles à la majorité des producteurs. Les bonnes années, une minorité de paysans pourra également se procurer

Figure 3. Revenu agricole et surplus par actif domestique en fonction des ressources consacrées à la captation de la force de travail entre mai et août $(n=58)$

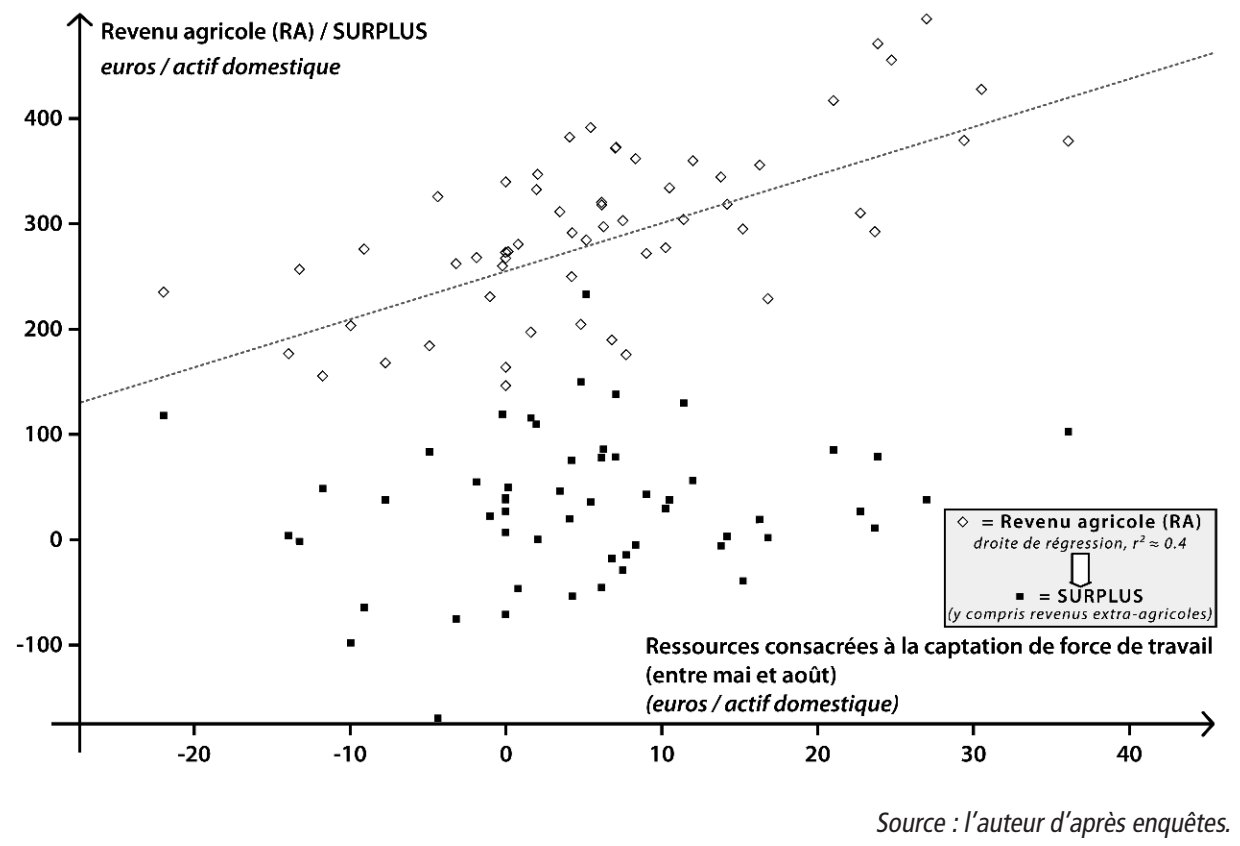


un petit ruminant (entre 30 et $40 €$ ) et (re) constituer peu à peu un troupeau. Une fois atteint un certain âge, un « chef de maison » se doit d'avoir un toit en tôles ondulées. Une telle maison revient environ à $360 €$. Dans ce but, les paysans forment des tontines et épargnent 5 à 10 ans. L'acquisition d'une moto $125 \mathrm{cc}$ (plus de $900 €$ ) est hors de portée de l'immense majorité des paysans.

Les unités de production/consommation pour lesquelles nous avons calculé un niveau de surplus négatif (jusqu'à - $90 € /$ an/actif domestique) peuvent passer une année exceptionnellement difficile grâce à la solidarité des réseaux de parenté et de voisinage. Mais lorsque le hiatus entre la capacité productive et le nombre de bouches à nourrir devient structurel, elles éclatent : les épouses divorcent et rejoignent avec leur progéniture un autre foyer, les enfants sont placés chez des oncles plus prospères, les jeunes actifs n'hésitent pas à fuir vers Freetown ou les districts diamantifères.

La faiblesse des surplus dégagés par les unités de production/consommation n'est pas un résultat surprenant dans le contexte d'une agriculture manuelle n'employant aucun intrant d'origine industrielle : la faiblesse de la productivité du travail se traduit mécaniquement par un faible surproduit dégagé par actif.

Plus surprenant est le fait que, contrairement au RA, les niveaux de surplus se sont pas corrélés aux ressources engagées dans la captation de journées de travail. Autrement dit, les unités de production/ consommation qui captent de la force de travail ne dégagent pas un surplus supérieur à celles qui en cèdent.

Cette situation s'explique par les disparités entre les unités de production/ consommation du point de vue du nombre de bouches à nourrir par actif domestique (figure 4). Sur l'ensemble des 58 cas d'étude, un actif domestique prend en charge en moyenne 1,9 consommateur, y compris lui-même. Mais dans certains groupes, il chute à moins de 1,5 (par exemple un couple avec 2 enfants) tandis que dans d'autres il grimpe au-delà de 2,5 (l'équivalent d'un couple avec 6 enfants).

Figure 4. Représentation de l'échantillon des unités de production/consommation en fonction du nombre de consommateurs par actif domestique

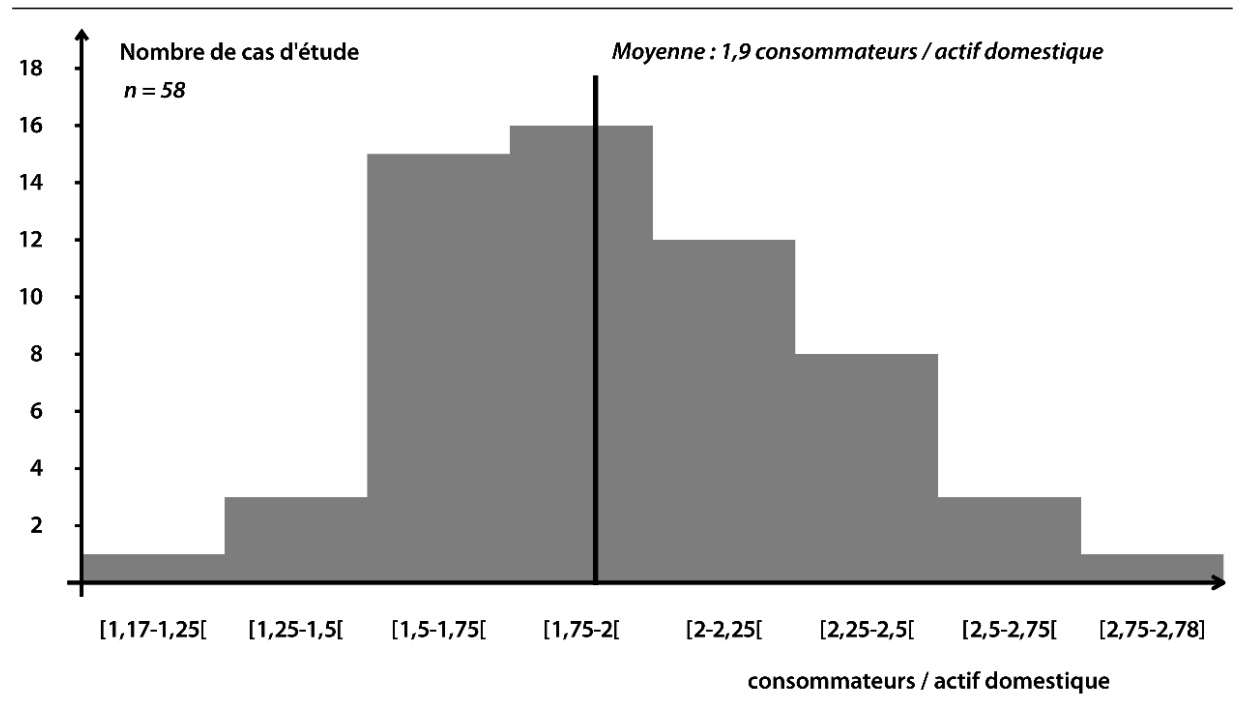

Source : I'auteur d'après enquêtes. 
Contrairement au modèle classique de Tchayanov (1990 [1925]), ces disparités ne reflètent pas le croît démographique « naturel » des foyers. Comme dans un grand nombre de sociétés paysannes (Lallemand, 1993), la circulation enfantine est aujourd'hui encore très importante en Sella Limba. Parmi les femmes, 148 ont été interrogées dans les études de cas. Sur leurs 223 rejetons encore à la charge d'un adulte, 61 (27\%) sont élevés par une autre femme. Inversement, les femmes interrogées élèvent 72 enfants $(30 \%$ des enfants à charge) dont elles ne sont pas les génitrices ${ }^{11}$.

Ainsi, si en plus de la part de la valeur ajoutée des unités de production/consommation consacrée à la captation de force de travail, on considère la part du revenu destinée à l'adoption et à la formation de pré-productifs, on constate que la disparité économique que l'on observe à l'échelle des cycles de culture ne se traduit pas par un processus de différenciation durable. La captation de la force de travail ne se cristallise pas dans l'accumulation de moyens de production supplémentaires ou plus performants, mais par la possibilité d'adopter et d'élever un plus grand nombre de préproductifs. Inversement, les pourvoyeurs de force de travail ne semblent pas décapitaliser ou se prolétariser au sein des villages, mais sont plutôt dans l'incapacité de prendre en charge leur propre progéniture.

La pratique est ancienne, mais la marchandisation de la force de travail modifie-telle son sens et sa portée ? Cette circulation correspond-elle à un mécanisme de régulation des inégalités ? Sans exclure toute dimension affective, nos informateurs décrivent moins l'adoption d'enfants comme un moyen d'aider ceux qui ne parviennent plus à subvenir aux besoins de leur progéniture que comme un investissement grâce

11. Cette situation n'est pas exceptionnelle en Sierra Leone (Bledsoe et Isiugo-Abanihe, 1989 ; Isaac et Conrad, 1982). auquel ils espèrent se retrouver à l'avenir moins démunis face à la précarité de l'existence. Si, en suivant Meillassoux (1975), on analyse l'économie des processus productifs sur une génération, on peut assimiler l'adoption d'enfants comme une stratégie de captation d'énergie de travail en formation à l'échelle viagère nourrie par la captation de force de travail à l'échelle des cycles annuels (Palliere et al., 2014). En reprenant le concept de «droit » comme « action socialement légitimée sur un bien » (Colin et Soro, 2008), on peut parler d' " accumulation de droits » sur l'énergie de travail d'autrui. De ce point de vue l'adoption des enfants, résultant de l'incapacité de certains individus à jouir de leur propre force de travail, serait plutôt une source d'inégalités au sein de la paysannerie. Cependant, l'accumulation d'énergie de travail via l'adoption d'enfants ne peut pas être assimilée à une accumulation de capital productif génératrice d'une différenciation durable. Elle reste une stratégie individuelle puisque les droits sur le travail d'autrui s'éteignent avec la mort de celui qui peut en jouir.

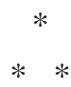

La marchandisation de la force de travail au sein des villages a été rendue possible par l'éclatement des grandes unités de production/consommation en unités de taille toujours plus réduite et structurellement plus fragiles, conjugué à la monétarisation du revenu des producteurs. Dans un contexte de stagnation, voire de régression, de la productivité du travail, en l'absence d'accumulation dans des moyens de production plus performants ou dans des ressources foncières, la captation de journées de travail extra-domestique apparaît comme une source potentielle de différenciation économique au sein de la paysannerie.

En lien avec cette analyse historique, l'évaluation des performances économiques des unités de production/consommation montre que ces échanges 
marchands de force de travail expliquent en grande partie les disparités importantes observées entre les revenus agricoles. Mais le niveau de revenu réellement disponible pour l'accumulation, une fois pris en compte les rémunérations extra-agricoles et le seuil de reproduction simple des unités de production/consommation, n'est pas corrélé à la capacité des producteurs à capter de la force de travail extra-domestique. On explique ce résultat par l'intense circulation enfantine entre les villages et les maisons.

La marchandisation de la force de travail, malgré les importantes disparités économiques entre capteurs et pourvoyeurs de force de travail, n'aboutit pas mécaniquement à une différenciation durable de la société paysanne comme le prévoit un schéma classique (Bernstein, 2010). La recherche des stratégies d'accumulation déployées par les paysans en Sella Limba nous conduit à dépasser le pas de temps du cycle agricole et à placer l'analyse à l'échelle viagère. Ce changement d'échelle nous invite à considérer non plus seulement la force de travail, qui circule sous forme de journées de travail entre les unités de production/consommation, mais également l'énergie de travail, inséparable de l'être social dans son intégralité et circulant entre les générations. Dans cette perspective, il faudrait intégrer à notre schéma l'énergie de travail formée par le secteur paysan qui lui échappe avec le départ, à chaque génération, de nombreux jeunes actifs pour les districts diamantifères et les villes $^{12}$.

L'auteur remercie Jean-Luc Paul pour ses critiques et ses commentaires qui ont nourri les analyses de cet article.

\section{RÉFÉRENCES BIBLIOGRAPHIQUES}

Bernstein H. (2010). Class Dynamics of Agrarian Change. Halifax, Fernwood Publishing, $142 \mathrm{p}$.

Binder K. (1989). The vicious cricle of hunger and indebtedness. Analysis of the farming system of South-East Bombali District, Sierra Leone, Wageningen, ICRA, 33 p.

Binns J. A. (1982). Agricultural change in Sierra Leone. Geography, vol. 67, $\mathrm{n}^{\circ} 2$, pp. 113-125.

Bledsoe C. H., Isiugo-Abanihe U. (1989). Strategies of Child Fosterage among mende Grannies in Sierra Leone. In Lesthaeghe R. (ed.), Reproduction and social organization in sub-Saharan Africa, Berkeley, University of California Press, pp. 442-474.

Chambers R., Conway G. R. (1992). Sustainable rural livelihoods: practical concepts for the 21st century. IDS discussion paper, $\mathrm{n}^{\circ} 296,29 \mathrm{p}$.

Chauveau J.-P. (2010). La loi de 1998 sur le domaine rural dans l'histoire des politiques foncières en Côte d'Ivoire. Une économie politique des transferts de droits entre 'autochtones' et 'étrangers' en zone forestière. In Colin J.-Ph., Le Meur P. Y., Léonard E., Les politiques d'enregistrement des droits fonciers. Du cadre légal aux pratiques locales, Paris, Karthala, pp. 105-140.

Chauveau J.-P., Richards P. (2008). West African Insurgencies in Agrarian Perspective : Côte d'Ivoire and Sierra Leone Compared. Journal of Agrarian Change, vol. 8, $\mathrm{n}^{\circ} 4$, pp. 515-52.

Chauveau J-.P., Colin J-.Ph., Lavigne Delville Ph., Le Meur P.-Y. (2006). Modes

12. Cette piste est poursuivie dans Palliere et al. (2014). 
d'accès à la terre, marchés fonciers, gouvernance et politiques foncières en Afrique de l'Ouest. Londres, IIED, 91 p.

Clarke J. I. (1969). Sierra Leone in maps. Londres, University of London Press, 116 p.

Cochet H. (2011). L'agriculture comparée. Versailles, Quae, 159 p.

Cochet H., Devienne S. (2006). " Fonctionnement et performances économiques des systèmes de production agricole : une démarche à l'échelle régionale ». Cahiers Agricultures, vol. 15, $\mathrm{n}^{\circ}$ 6, pp. 578-83.

Colin J.-Ph., Soro D. M. (2008). Marchandisation, individualisation et gestion intrafamiliale des droits sur la terre en basse Côte d'Ivoire. Économie rurale, n ${ }^{\circ}$ 303-304-305, pp. 154-168.

Couty Ph. (1983). Qualitatif et quantitatif. AMIRA, Qualitatif et quantitatif : deux modes d'investigation complémentaires. Paris, ORSTOM, pp. 35-47.

Delarue J. (2007). Mise au point d'une méthode d'évaluation systémique d'impact des projets de développement agricole sur le revenu des producteurs : étude de cas en région kpèlè (République de Guinée). Thèse de doctorat, Paris, AgroParisTech, 509 p.

Deveneaux G. H. K. (1973). The political and social impact of the colony in the Northern Sierra Leone 1821-1896. Boston, Boston University Press, 439 p.

Gastellu J.-M. (1980). Mais, où sont donc ces unités économiques que nos amis cherchent tant en Afrique ? Cahiers ORSTOM, Série Sciences humaines, vol. 17, $\mathrm{n}^{\circ}$ 1-2, pp. 3-11.

Gomez y Paloma S., Acs S., Saravia Matus S., Lakoh A., Baudoin M., Hites G., Sammeth F. (2012). Rural poverty reduction and food security: The case of smallholders in Sierra Leone. Joint Research Centre Report, Seville, European Commission, 213 p.

Isaac B. L., Conrad S R. (1982). Child fosterage among the Mende of Upper Bambara chiefdom, Sierra Leone: rural-urban and occupational comparisons. Ethnology, vol. 21, $\mathrm{n}^{\circ} 3$, pp. 243-257.

Jolivel M., Palliere A. (2007). Diagnostic agraire d'une petite région agricole au Nord-Ouest de la Sierra Leone. Mémoire de DAA, INA-PG, AgroParisTech, 67 p.
Jouve Ph. (2006). Transition agraire : la croissance démographique, une opportunité ou une contrainte ? Afrique contemporaine, n²17, pp. 43-54.

Lallemand S. (1993). La circulation des enfants en société traditionnelle : prêt, don, échange. Paris, L'Harmattan, 223 p.

Leach M. (1994). Rainforest Relations: Gender and Resource Use Among the Mende of Gola, Sierra Leone. Edinburgh, Edinburgh University Press, 308 p.

Little K. L. (1948). The Mende Farming Household. The Sociological Review, vol. 40, $\mathrm{n}^{\circ} 1$, pp. 37-56.

Maconachie R., Binns T. (2007). Farming miners or mining farmers?: Diamond mining and rural development in post-conflict Sierra Leone. Journal of Rural Studies, vol. 23, $\mathrm{n}^{\circ} 3$, pp. 367-80.

MAFFS (2009). National Sustainable Agriculture Development Plan 2010-2030. Freetown, Government of Sierra Leone, $41 \mathrm{p}$.

MAFFS(2010).Smallholder Commercialisation Programme. Investment Plan. Freetown, Government of Sierra Leone, $32 \mathrm{p}$.

Mazoyer M., Roudart L. (1997). Histoire des agricultures du monde: du néolithique à la crise contemporaine. Paris, Seuil, 533 p.

McCulloch M. (1950). The Peoples of Sierra Leone Protectorate. Londres, International African Institute, 102 p.

Meillassoux C. (1975). Femmes, greniers et capitaux. Paris, Maspero, $254 \mathrm{p}$.

Meillassoux C. (1977). Élaboration d'un modèle socio-économique en ethnologie. Terrains et théories, Paris, Anthropos, pp. 69-77.

Migeod F. W. H. (1926). A view of Sierra Leone. London, Paul, Trench \& Trubner, 351 p.

Milleville P. (1972). Approche agronomique de la notion de parcelle en milieu traditionnel africain : la parcelle d'arachide en moyenne-Casamance. Cahiers ORSTOM. Série Biologie, $\mathrm{n}^{\circ}$ 17, pp. 23-37.

Palliere A. (2008). Pratiques paysannes et dynamiques agraires à l'échelle d'un territoire. Sella Limba (Sierra Leone). Mémoire de DAA, AgroParisTech, $340 \mathrm{p}$. 
Palliere A. (2013). Au-delà de Malthus et Boserup : comprendre les dynamiques agro-écologiques d'un territoire. Un cas d'étude en Sella Limba, Sierra Leone. Paris, Rencontre JCEA, 11-12 janvier 2013.

Palliere A. (2014). Un sac de riz vide ne tient pas debout. Dynamiques agraires régionales et marginalisation de la paysannerie sierraléonaise. Thèse de doctorat, Nanterre, Université Paris Ouest Nanterre La Défense, $572 \mathrm{p}$.

Palliere A., Paul J.-L., Cochet H., Landy F. (2014). Marchandisation du travail et différenciation sociale en Sella Limba (Sierra Leone) de 1950 à nos jours. Journal des Africanistes, vol. 83, $\mathrm{n}^{\circ}$ 2, pp. 116-141.

Passeron J-C. (2006). Le raisonnement sociologique : un espace non poppérien de l'argumentation. Paris, Albin Michel, 666 p.

Paul J.-L. (2003). Anthropologie historique des Hautes Terres de Tanzanie orientale. Paris, Karthala, 342 p.

Paul J.-L. (2008). Au-delà de Femmes, Greniers et Capitaux. Journal des anthropologues, $\mathrm{n}^{\circ}$ 114-115, pp. 223-245.

Phelinas P. (1986). Politique des prix du riz, incitation à la production et effet sur la répartition des revenus dans six pays africains. Paris, ORSTOM, 383 p.

Reno W. (1995). Corruption and state politics in Sierra Leone. Cambridge, Cambridge University Press, 229 p.

Richards P. (1983). Ecological Change and the Politics of African Land Use. African Studies Review, vol. 26, $\mathrm{n}^{\circ} 2$, pp. 1-72.

Richards P. (1985). Indigenous agricultural revolution: ecology and food production in West Africa. Londres, Westview Press, $192 \mathrm{p}$.
Richards P. (1996). Fighting for the Rain Forest : War, Youth \& Resources in Sierra Leone. Londres, International African Institute, $216 \mathrm{p}$.

Richards P., Bah K., Vincent J. (2004). Social capital and survival : prospects for community-driven development in post-conflict Sierra Leone. Washington, Banque mondiale, $78 \mathrm{p}$.

Riddell J. B., (1970). The spatial dynamics of modernization in Sierra Leone: structure, diffusion, and response. $\mathrm{PhD}$ Thesis, Evanston, Northwestern University Press, 142 p.

Sébillotte M. (1977). Jachère, système de culture, système de production, méthodologie d'étude. Journal d'Agriculture traditionnelle et de Botanique appliquée, vol. 24, $\mathrm{n}^{\circ}$ 2-3, pp. 241-264.

Scoones I. (2009). Livelihoods perspectives and rural development. The Journal of Peasant Studies, vol. 36, n 1, pp. 171-196.

Statistics Sierra Leone (2006). 2004 рориlation and housing census. Final Results. Freetown, Government of Sierra Leone.

Swindell K. (1974). Sierra Leonean Mining Migrants, Their Composition and Origins. Transactions of the Institute of British Geographers, n61, pp. 47-64.

Tchayanov A. V. (1990 [1925]). L'organisation de l'économie paysanne. Paris, Éditions de la Maison des sciences de l'homme, 344 p.

Zack-Williams A. B. (1982). Merchant Capital and Underdevelopment in Sierra Leone. Review of African Political Economy, n ${ }^{\circ} 25$, pp. 74-82.

Zack-Williams A. B. (1999). Sierra Leone: The Political Economy of Civil War, 1991-98. Third World Quarterly, vol. 20, $\mathrm{n}^{\circ} 1$, pp. 143-62. 